\title{
BMJ Open Ratio of asymptomatic COVID-19 cases among ascertained SARS-CoV-2 infections in different regions and population groups in 2020: a systematic review and meta-analysis including 130 123 infections from 241 studies
}

To cite: Chen X, Huang Z, Wang J, et al. Ratio of asymptomatic COVID-19 cases among ascertained SARS-CoV-2 infections in different regions and population groups in 2020: a systematic review and meta-analysis including 130123 infections from 241 studies. BMJ Open 2021;11:e049752. doi:10.1136/ bmjopen-2021-049752

- Prepublication history and additional supplemental material for this paper are available online. To view these files, please visit the journal online (http://dx.doi.org/10.1136/ bmjopen-2021-049752)

$\mathrm{XC}, \mathrm{ZH}$ and JW contributed equally.

$\mathrm{XC}, \mathrm{ZH}$ and JW are joint first authors.

Received 08 February 2021 Accepted 15 0ctober 2021

Check for updates

(c) Author(s) (or their employer(s)) 2021. Re-use permitted under CC BY-NC. No commercial re-use. See rights and permissions. Published by BMJ.

For numbered affiliations see end of article.

\section{Correspondence to}

Jingxuan Wang;

jxwang@link.cuhk.edu.hk and

Dr Jinhui Li;

clairehh4588@gmail.com

\section{ABSTRACT}

Introduction Asymptomatic infection of SARS-CoV-2 may lead to silent community transmission and compromise the COVID-19 pandemic control measures. We aimed to estimate the rate of asymptomatic COVID-19 from published studies and compare this rate among different regions and patient groups.

Methods In this systematic review and meta-analysis, electronic databases including Medline, Embase, PubMed and three Chinese electronic databases (Chinese National Knowledge Infrastructure [CNKI], WanFang Data and China Science, and Technology Journal Database [VIP]) were searched for literature published from 1 November 2019 to 31 December 2020. Original investigations with sample size (or number of subjects) not less than five were included for further analyses. Subgroup analyses were conducted according to different study types, study periods, geographical regions and patient demographics. The STATA (V.14.0) command 'metaprop' was implemented to conduct a meta-analysis of the pooled rate estimates of asymptomatic infections with exact binomial and score test-based 95\% confidence interval $(\mathrm{Cl})$.

Results A total of 130123 ascertained COVID-19 infections from 241 studies were included in this metaanalysis, including 31411 asymptomatic infections. The overall rate of asymptomatic infections was $23.6 \%$ (18.5\%-29.1\%) and 21.7\% (16.8\%-27.0\%) before and after excluding presymptomatic cases, respectively. Subgroup analysis showed that significantly higher in pregnant women $(48.8 \%, 28.9 \%-68.9 \%)$, children $(32.1 \%, 24.2 \%-40.5 \%)$, and studies reporting screening programmes $(36.0 \%, 24.6 \%-48.1 \%)$ conducted on or after 1 March 2020 (42.5\%, 33.4\%-51.9\%). In terms of geographical region, the rate was the highest in Africa $(64.3 \%, 56.7 \%-71.6 \%)$, followed by America $(40.0 \%$, $27.4 \%-53.3 \%)$, Europe $(28.1 \%, 19.0 \%-38.1 \%)$ and Asia (18.1\%, 13.2\%-23.5\%).

Conclusion We approximated that one-fifth of COVID-19 infections are asymptomatic throughout the course of infection. Public health policies targeting these highrisk groups may be recommended to achieve early
Strengths and limitations of this study

- The comprehensive systematic literature search included a greater number of studies which reported varied rates of asymptomatic infections.

- Several subgroup analyses were conducted by considering different aspects of the study design.

- Heterogeneity in rates of asymptomatic infections shall be noted.

- The cross-sectional design of most reviewed studies may misclassify 'pre-symptomatic' as 'symptomatic' infections.

identification and more stringent containment of the pandemic.

\section{INTRODUCTION}

In 2019, a cluster of severe pneumonia cases of unknown type were reported in Wuhan, China. ${ }^{1}$ Later coined as COVID-19, it rapidly resulted in large-scale outbreaks across many regions. On 30 January 2020, the WHO declared COVID-19 as a public health emergency of international concern ${ }^{2}$ and further defined it as a pandemic on 11 March 2020. As of 29 October 2021, a cumulative total of 245373039 COVID-19 cases have been confirmed globally, with 4979421 associated deaths.

The pathogen of COVID-19, that is, SARSCoV-2, is highly contagious and could be transmitted from human to human. ${ }^{3}$ The viral load of an asymptomatic patient has been found to be similar to that of a symptomatic patient in a study of nine patients, ${ }^{4}$ and this observation was later confirmed in a study involving large samples. ${ }^{5}$ The early 
peaking of SARS-CoV-2 viral load during the presymptomatic phase may cause silent community outbreaks. ${ }^{6}$ Further investigations also found that asymptomatic infections may carry SARS-CoV-2 for more than 1 month, indicating the long-lasting risk of secondary infection. ${ }^{7-11}$

Determining the rate of asymptomatic is important as it may deepen the understanding of the real reproductive number $\left(R_{0}\right)$, as well as the true incidence and mortality rate of COVID-19. The rates may also function as an essential epidemiological parameter to inform disease-combating policies, including the density and range of screening, patient isolation, as well as early intervention. ${ }^{12}$ Nevertheless, the significance of the invisibly infected person as the source of infection depends on the distribution in the population and on the amount and duration of the virus excreted. ${ }^{13}$ The reported proportions of asymptomatic individuals in existing literature varied a lot, ${ }^{12}$ depending on research setting (e.g., geographical regions, screening vs non-screening studies), demographic characteristics (e.g., age groups, pregnant women, children) and other latent factors. A systematic review and meta-analysis was performed to estimate the rate of asymptomatic infections based on published studies and compare the rate among different patient groups as well as study settings.

\section{METHODS}

\section{Search strategy}

This systematic review and meta-analysis was conducted strictly following the 'Preferred Reporting Items for Systematic Reviews and Meta-Analyses' guideline. ${ }^{14} \mathrm{XC}$ and $\mathrm{ZH}$ searched Medline, Embase, PubMed and the three most commonly used Chinese electronic databases (Chinese National Knowledge Infrastructure [CNKI], WanFang Data, and China Science and Technology Journal Database [VIP]) from 1 November 2019 to 31 December 2020. CNKI, WanFang Data and VIP are affiliates of the Chinese Ministry of Science and Technology, which provides access to peer-reviewed, continuously updated research journal articles in Chinese. The search string related to 'COVID-19' and 'asymptomatic' was systematically developed in PubMed using Medical Subject Headings (MeSH) terms and was applied to all databases after discussion with an experienced librarian (Maggie Choi). The 'Text Word' search field was used to ensure the best possible search evidence (online supplemental table 1). No filters or limitations were applied to retrieve the best possible results and to avoid excluding preindexed materials. Meanwhile, highly relevant references were also searched (by $\mathrm{XC}$ and $\mathrm{ZH}$ ) by reviewing the reference list of the included articles. Two reviewers (XC and $\mathrm{ZH}$ ) independently determined eligible studies. Consensus was reached by referral to a third reviewer (JW) when there was disagreement. All manuscripts were imported into the EndNote software (V.X8; Thomson Reuters, Carlsbad, California) to store and manage the retrieved citations. Duplicate studies were removed.

\section{Asymptomatic case and asymptomatic ratio}

Several similar definitions of 'asymptomatic case' were noted in previous studies. ${ }^{15}$ We followed the official definition from the State Council of China and the WHO, ${ }^{16} 17$ defining 'asymptomatic case' as individuals who (1) have no clinical manifestations of COVID-19, such as fever, cough, sore throat and other self-perceived or clinically identifiable symptoms and signs; (2) have positive result for SARS-CoV-2 pathogen test; and (3) do not develop symptoms until the end of hospital admission or follow-up observations. Asymptomatic COVID-19 infection rate, or asymptomatic ratio, is calculated as the proportion of asymptomatic cases among all COVID-19 infections.

\section{Literature screening and selection criteria}

All studies were screened first by title and abstract, followed by full text if the study met the inclusion criteria, which consist of the following: (1) the subjects of the study were diagnosed with SARS-CoV-2 infection; (2) the study was designed as an observational study; and (3) the number of asymptomatic and symptomatic COVID-19 infections was explicitly and exactly reported.

Literature screening was conducted without language or region restrictions. The exclusion criteria were as follows: (1) the study included patients without virological evidence of SARS-CoV-2 infection; (2) the study did not investigate the distribution of asymptomatic COVID-19 infections among all subjects; (3) the study was not classified as original research, such as reviews, comments and case reports; and (4) the study had an overall sample size of less than five.

If the same group of subjects was reported by different articles, only articles with the most updated and detailed information were included for further analysis.

\section{Data extraction and subgrouping schemes}

For eligible articles, two types of information were independently extracted by two reviewers (XC and $\mathrm{ZH})$ : (1) basic information of individual studies, including name of the first author, investigation period, geographical region, sample size and characteristics of the subjects; and (2) the number of COVID-19 infections, including the total number of test-positive subjects, the number of presymptomatic infections and that of asymptomatic infections.

To ensure accuracy of data, cross-checking was conducted after extraction of the preliminary information. Disagreements were resolved through consensus or by referral to a third reviewer (JW).

To explore the source of heterogeneity among the included studies, several subgroup analyses were performed according to study design and characteristics of subjects in each study. First, we identified three groups based on subjects' demographic features: the 'children' group, consisting of subjects less than 18 years old; the 'pregnant women' group, involving expectant mothers as subjects; and the 'general population' group. Second, three age groups were identified according to subjects' 


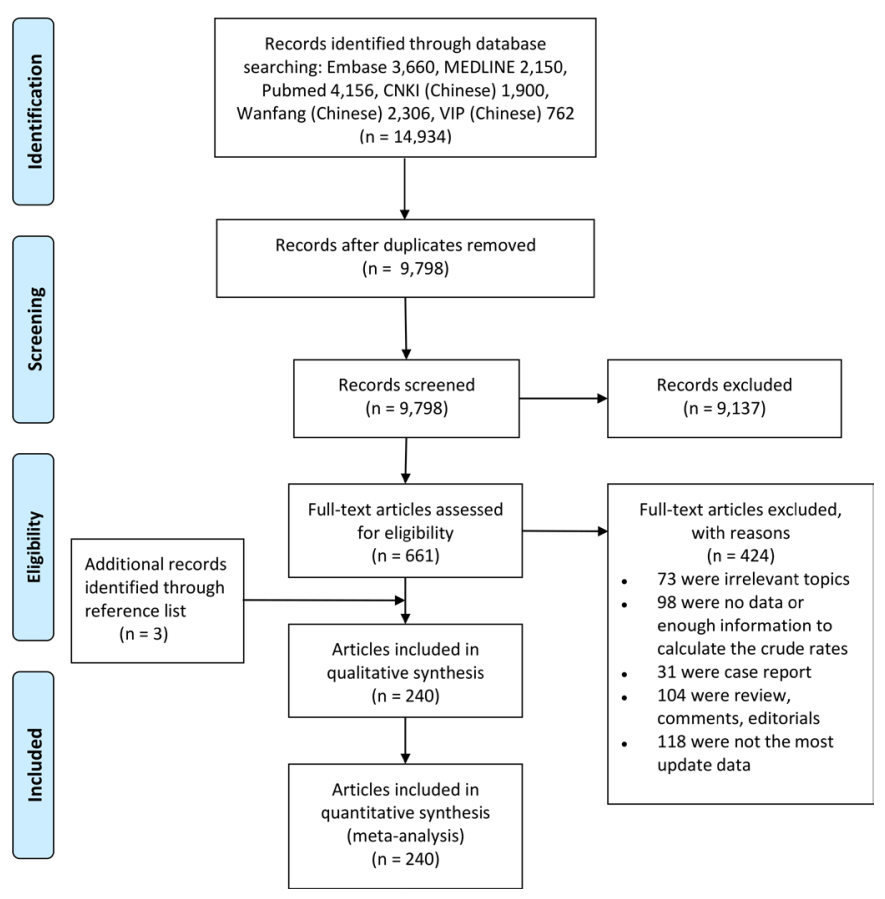

Figure 1 Study selection process for meta-analysis. CNKI, Chinese National Knowledge Infrastructure; PRISMA, Preferred Reporting Items for Systematic Reviews and MetaAnalyses.

mean or median age: $\leq 18$ years, $19-45$ years and $>45$ years. The selection of the mean or median age followed the statistics reported in each study. Third, studies were categorised into 'screening' or 'non-screening' by referring to the positive rate of SARS-CoV-2 pathogen test among the included subjects. In the screening studies, the positive rate was less than $100 \%$, while for nonscreening studies all subjects were tested positive. Fourth, we separated studies based on subjects from different geographical regions, including Africa, America, Asia and Europe. Last, subgroup analysis was performed by study period during which the subjects were tested for COVID19, including 'before 01 March 2020' or '01 March 2020 and afterwards'.

\section{Quality assessment}

The quality of each included study was independently assessed by two researchers (XC and $\mathrm{ZH}$ ) using the Appraisal tool for Cross-Sectional Studies (AXIS tool) ${ }^{18}$ which has 5 components including 20 questions. Seven questions measure the quality of reporting, another seven questions measure the quality of study design, and six questions measure the possible introduction of biases in each study.

\section{Statistical analysis}

Accounting for all included studies, pooled estimates of asymptomatic rates were generated with exact binomialdistributed likelihood framework and score test-based 95\% CI. ${ }^{1}$ The STATA (V.14.0) command 'metaprop' was adopted to conduct the meta-analysis. Heterogeneity across the studies was examined using $I^{2}$ statistics, measuring the proportion of total variation contributed by between-study variation. $I^{2}$ values $<25 \%, 25 \%-75 \%$ and $>75 \%$ correspond to the thresholds for three ordinal levels of heterogeneity, namely low, moderate and high, respectively. ${ }^{19}$

Univariate and multivariate meta-regression analyses were performed to identify any potential effects of modifiers or confounders on the estimated rate. We examined the effects of covariates including study population, age-specific proportion, screening/non-screening study, geographical region and time trend in the multivariate analysis. Sensitivity analysis was conducted by omitting one study at a time, generating pooled estimates and comparing with the original estimates. Potential publication bias was examined by Egger's test and visualised using a funnel plot. If the tests indicated potential publication bias, the trim and fill's method, which is based on a modified funnel plot, was adopted to adjust for smallstudy effects. ${ }^{20}$

All analyses were performed using STATA statistical software (V.14.0). The figures were generated using $\mathrm{R}$ software (V.3.6.3) with the 'forestplot' package.

\section{RESULTS}

\section{Characteristics of studies and subjects}

In total, 9798 unique citations were identified by the literature search in different databases (figure 1). We screened and assessed the eligibility of 661 full-text articles after 9137 citations were excluded during title or abstract screening using predetermined criteria. Finally, there were 241 studies in 240 articles included in the meta-analysis, among which 3 studies were additional records identified through reference lists.

The basic characteristics of the included studies are shown in online supplemental table 2. The characteristics include cities, countries and study periods when patients with ascertained COVID-19 infections were recruited; the number of positive tests and asymptomatic infections; the number of presymptomatic infection in patients if available; and the demographics of study subjects. Approximately $72.9 \%$ (175 out of 241) of all included studies were conducted in Asia, $11.7 \%$ in Europe and 10.42\% in America. In terms of study population, $80.5 \%$ were performed in general adults, $12.4 \%$ in children and $7.1 \%$ in pregnant women.

Quality scores of AXIS for the included studies ranged between 11 and 19 points, with 153 studies meeting the criteria for high quality ( $\geq 16$ points) (see online supplemental table 3 for details). Overall, 97.9\% (235 out of 240) of all studies met the criteria for both reporting the aims/objectives and quality design, and the risk factors and outcome variables were appropriate for the studies.

\section{Asymptomatic ratio estimates}

A total of 130123 ascertained COVID-19 infections from 241 studies were finally included in the meta-analysis 


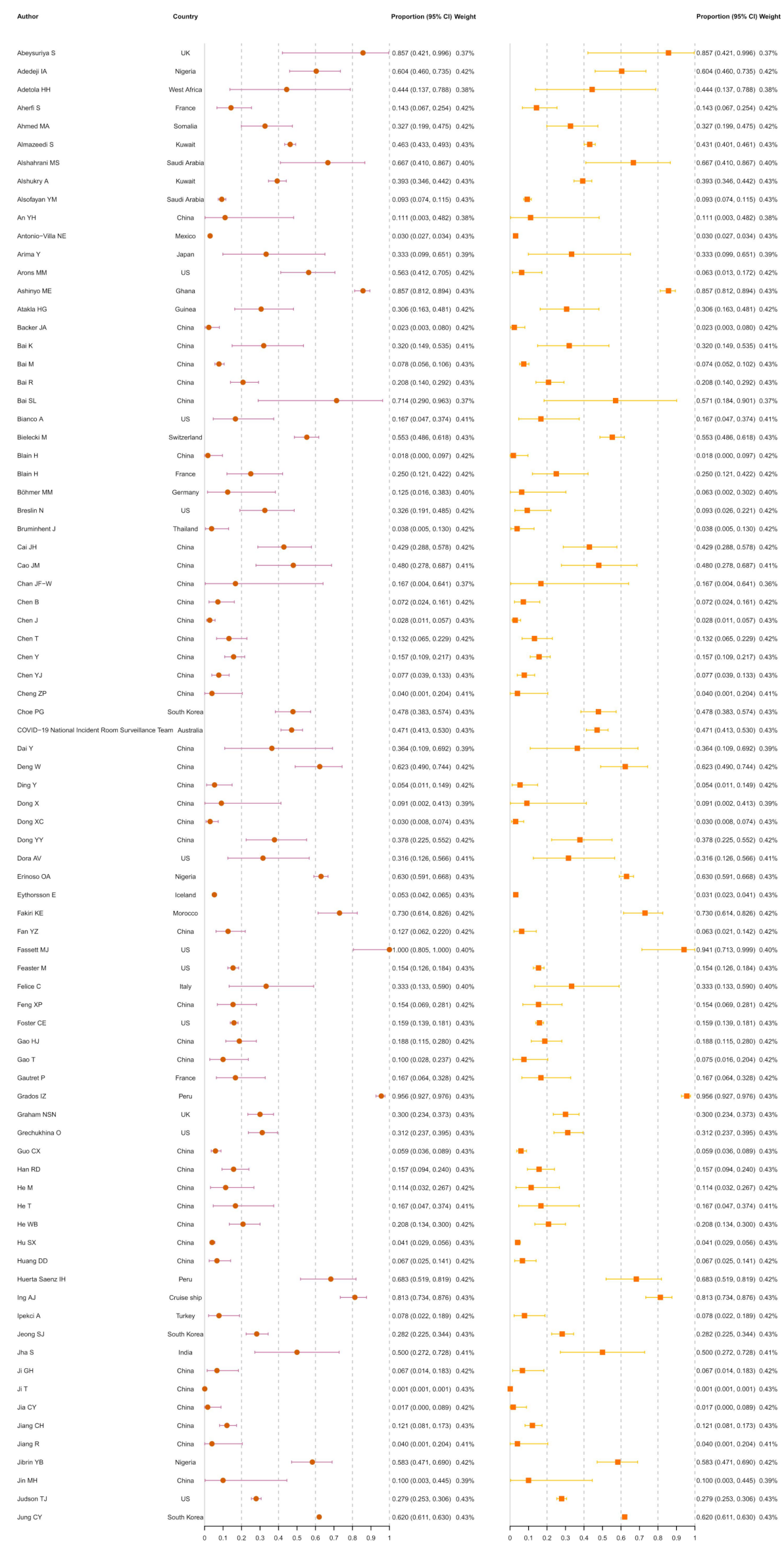

Figure 2 (Continued) 


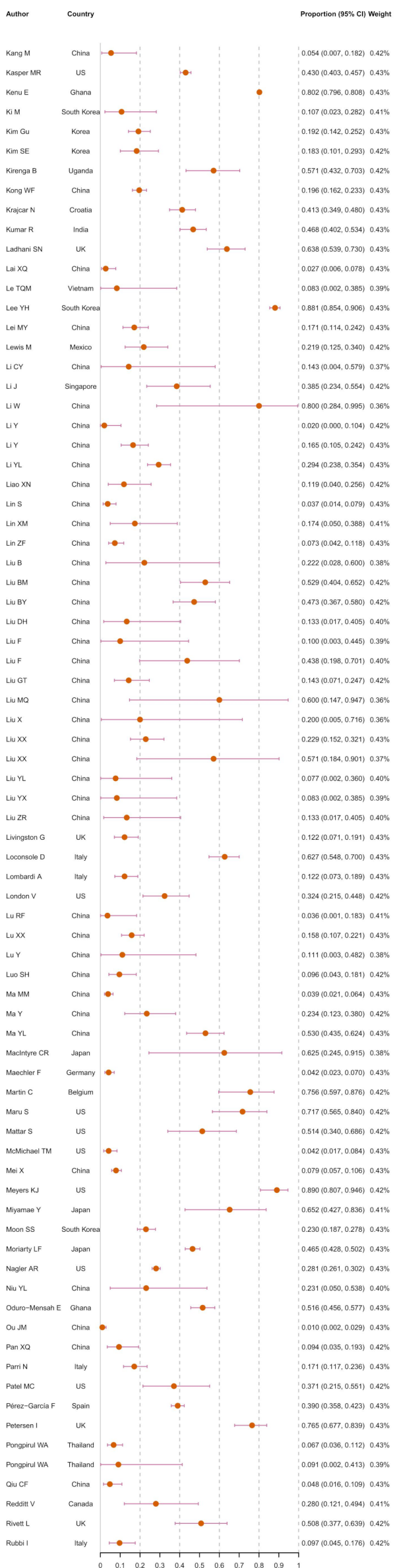

Figure 2 (Continued)

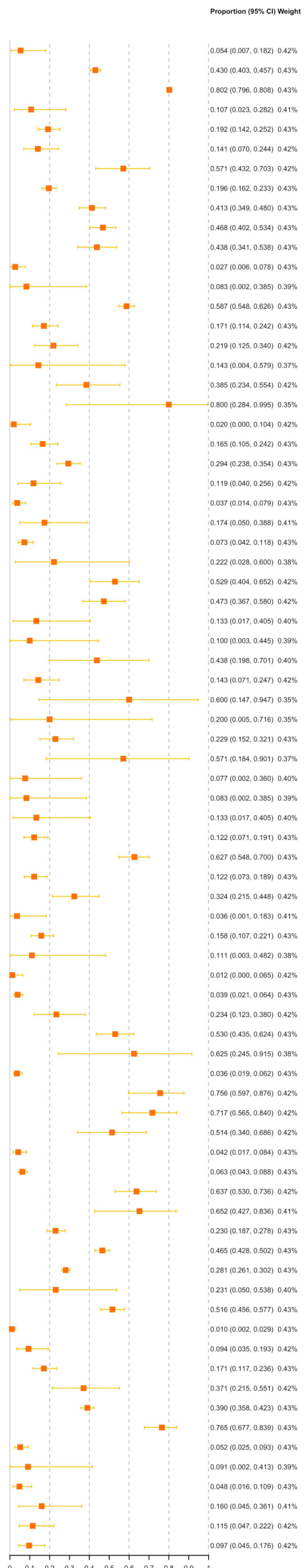




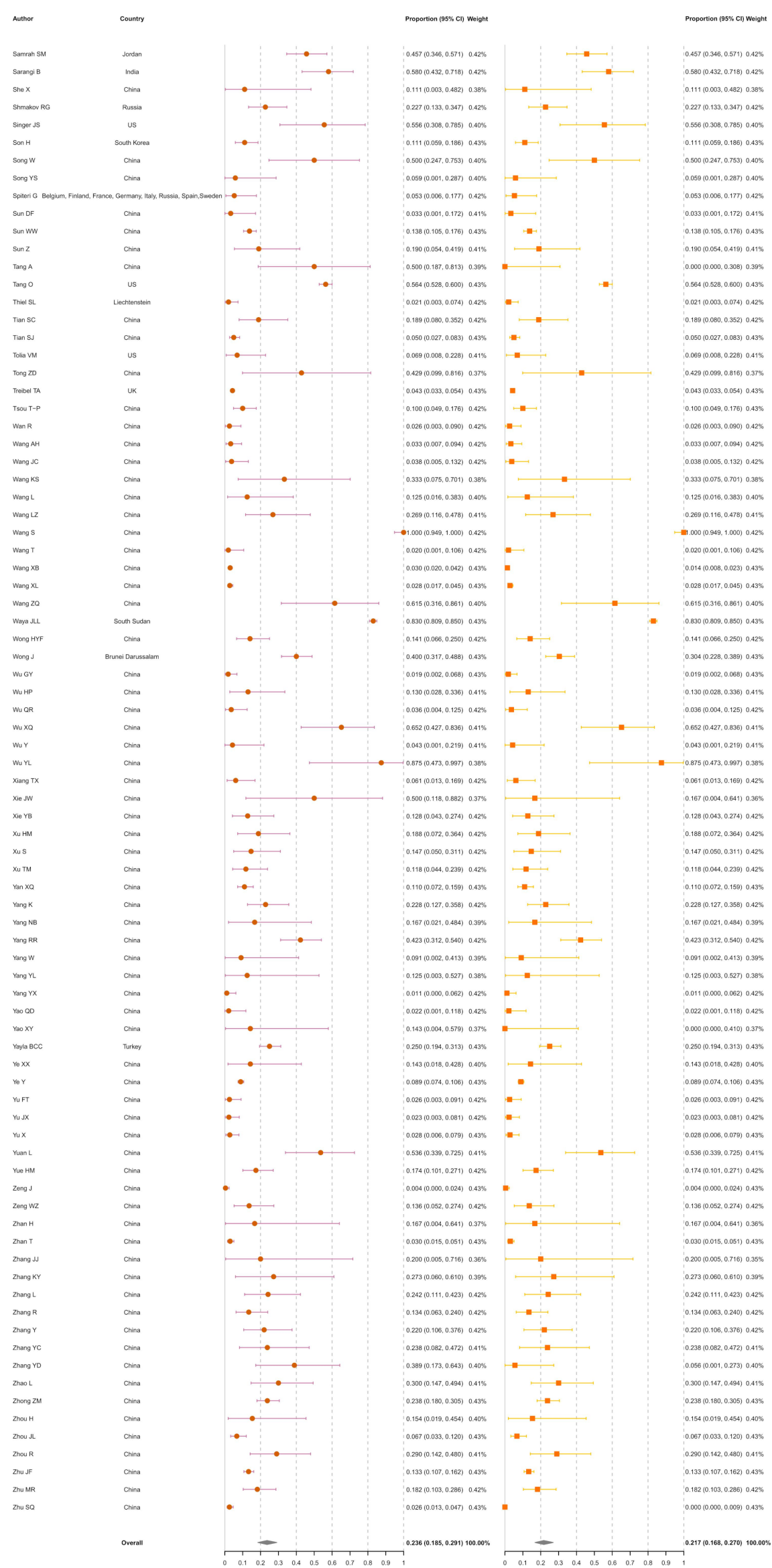

Figure 2 Meta-analysis of the asymptomatic ratio among ascertained COVID-19 infections (including and excluding presymptomatic infections). 
(figure 2), including 31411 asymptomatic infections at the time of diagnosis. The rate of asymptomatic infections varied from $0.1 \%$ in general adults in Wuhan, China, ${ }^{21}$ to $95.6 \%$ in pregnant women in Peru. ${ }^{22}$ The overall rate of asymptomatic infections was estimated at $23.6 \%(18.5 \%-$ $29.1 \%$ ). The $I^{2}$ equalled $99.7 \%$, indicating high heterogeneity among the studies. Of the 31411 asymptomatic infections, 448 (1.4\%) developed symptoms after admission. After excluding these presymptomatic observations, the pooled rate was $21.7 \%(16.8 \%-27.0 \%)$ with an $I^{2}$ of $99.7 \%$.

In a subgroup analysis, we found that pregnant women $\left(48.8 \%, 28.9 \%-68.9 \% ; \quad I^{2}=96.9 \%\right)$ had a significantly higher rate of asymptomatic infections than children $\left(32.1 \%, 24.2 \%-40.5 \% ; I^{2}=94.4 \%\right)$, whereas the general non-pregnant adults had the lowest asymptomatic rate $\left(20.4 \%, 15.0 \%-26.3 \% ; I^{2}=99.8 \%\right)$. Regarding age-specific proportion, patient groups with a mean/median age of $\leq 18$ years $\left(31.1 \%, 23.5 \%-39.2 \% ; I^{2}=94.4 \%\right)$ had a significantly higher prevalence of asymptomatic cases than those with a mean/median age of $19-45$ years $(26.7 \%$, $\left.19.1 \%-34.9 \% ; I^{2}=99.7 \%\right)$ or $>45$ years $(15.3 \%, 10.7 \%-$ $20.6 \% ; I^{2}=97.1 \%$ ).

The rate of asymptomatic infections was $36.0 \%$ $\left(24.6 \%-48.1 \% ; I^{2}=99.8 \%\right)$ among studies with screening setting, which was almost twice that among studies with non-screening setting $\left(19.4 \%, 14.3 \%-24.9 \% ; I^{2}=99.6 \%\right)$. Significantly different asymptomatic rates were observed in different geographical regions $(\mathrm{p}<0.001)$ - the rate was highest in Africa $\left(64.3 \%, 56.7 \%-71.6 \% ; I^{2}=96.8 \%\right)$, followed by America $\left(40.0 \%, 27.4 \%-53.3 \% ; I^{2}=99.6 \%\right)$ and Europe $\left(28.1 \%, 19.0 \%-38.1 \% ; I^{2}=98.5 \%\right)$. Asia was estimated to have the lowest rate of asymptomatic infections $\left(18.1 \%, 13.2 \%-23.5 \% ; I^{2}=99.5 \%\right)$. In the subgroup analysis by time period, we noted that studies conducted before 1 March 2020 reported a pooled asymptomatic infection rate of $13.3 \%\left(9.8 \%-17.1 \% ; I^{2}=94.2 \%\right)$, while the rate increased sharply to $42.5 \%\left(33.4 \%-51.9 \% ; I^{2}=99.6 \%\right)$ among studies conducted on or after 1 March 2020.

The results of all subgroup analyses were shown in figure 3 .

We performed both univariate and multivariate metaregression analyses to investigate study-level factors that may have contributed to the heterogeneity among studies and might have influenced our estimations of the rate of asymptomatic infections. The results of univariate regression analysis (table 1) demonstrated several significant sources of heterogeneity in the estimated rate of asymptomatic infections-studies focused on subjects aged $>45$ years old $(p=0.015)$ and conducted out of Africa $(p \leq 0.001)$ were significantly associated with a lower ratio of asymptomatic infections, whereas those using a screening study design $(\mathrm{p}<0.001)$ and conducted on or after 1 March $2020(p<0.001)$ tended to report a higher ratio of asymptomatic infections. A screening study design $(\mathrm{p}=0.031)$, geographical region $(\mathrm{p}<0.001)$ and time trend $(\mathrm{p}<0.001)$ remained as significant sources of heterogeneity in the multivariate regression analysis.

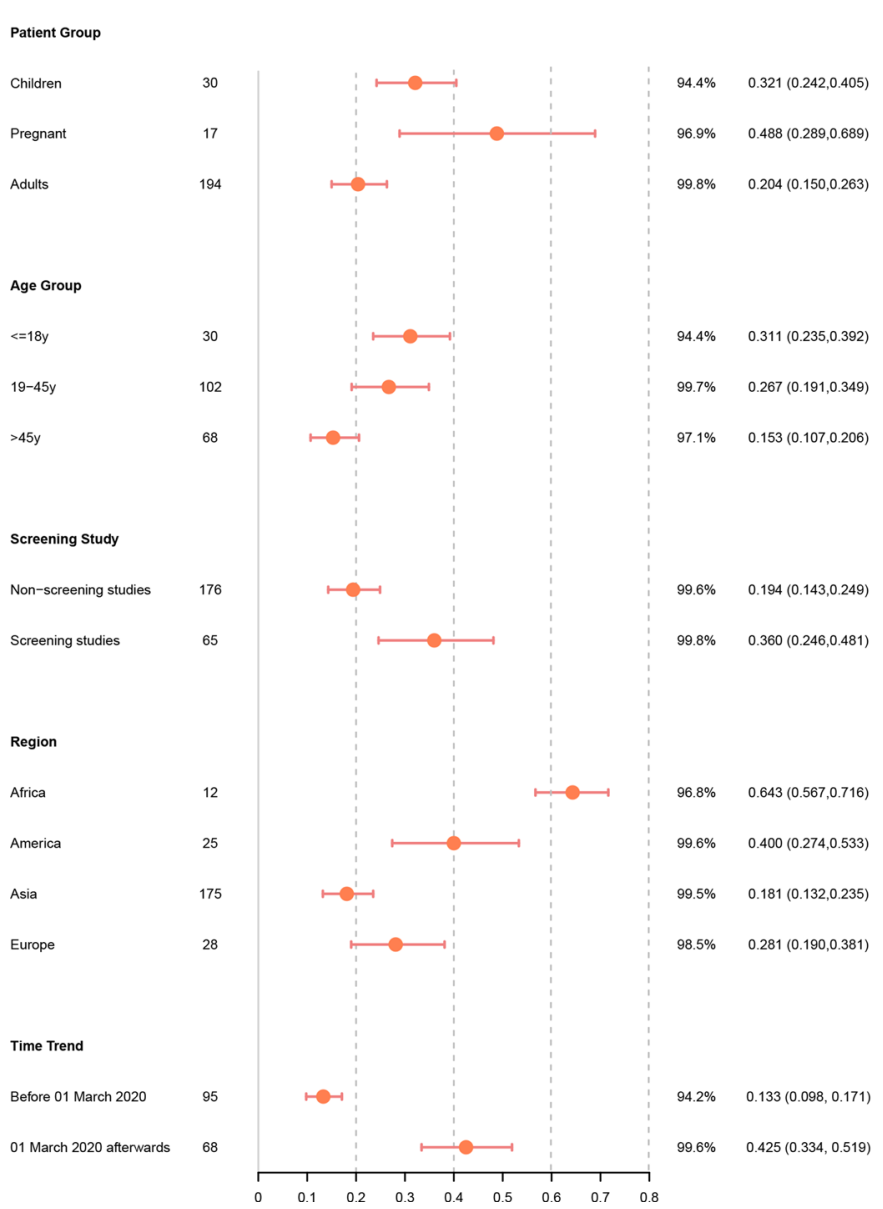

Figure 3 Asymptomatic ratio among ascertained COVID-19 infections by patient group, age group, screening study, region and time trend.

We found no evidence of publication bias among studies by Egger's test with $p=0.414$. Sensitivity analysis showed that it was unlikely that any individual study significantly influenced the pooled estimates, demonstrating the robustness and reliability of our estimates (online supplemental table 4).

\section{DISCUSSION}

Our meta-analysis of 130123 infections in 241 studies performed in 36 countries provides an up-to-date as well as a comprehensive overview of the asymptomatic infection rate of COVID-19. The estimated rate in our study were $23.6 \%$ and $21.7 \%$ before and after excluding presymptomatic cases, respectively. There were three key findings in the subgroup analysis. ${ }^{23}$ First, African studies reported the highest asymptomatic infection rate, while Asian studies reported the lowest. Second, the proportion of asymptomatic carriers was nearly doubled in screening studies than in non-screening studies. Third, asymptomatic infection rate was more than two times higher in studies conducted on or after 1 March 2020 than those conducted beforehand. 
Table 1 Results of univariate and multivariate meta-regression analyses

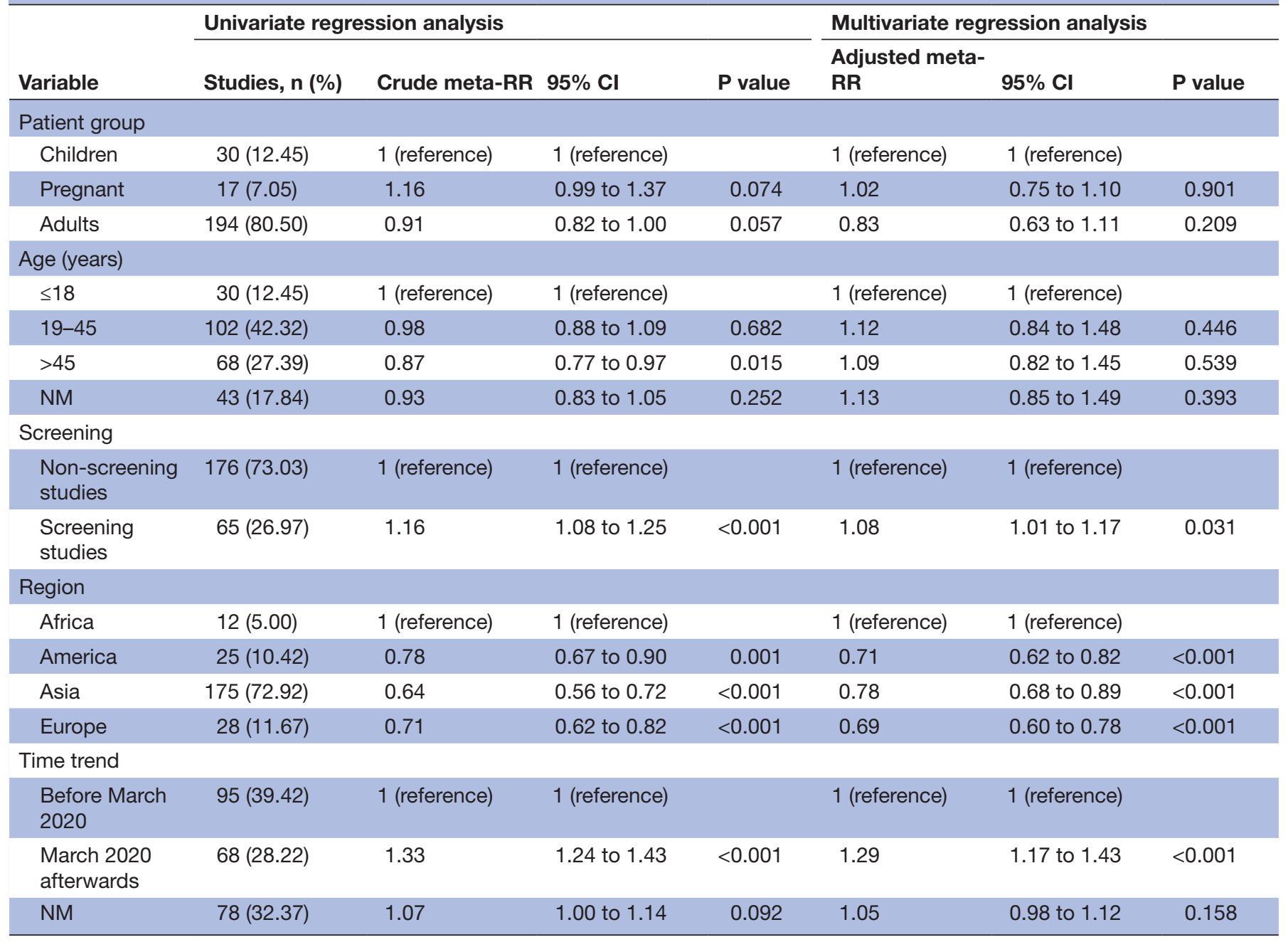

$\mathrm{NM}$, not mentioned; RR, risk ratio.

Our estimation is higher than some existing metaanalyses, which reported a ratio between $15.6 \%$ and $20 \% .^{24}{ }^{25}$ One possible explanation is that the included studies in previous meta-analyses were dominated by that conducted during the early course of the pandemic and in China or the USA, where the asymptomatic ratio was lower than other regions. However, our study included more than 200 articles, resulting in a more precise estimation with significantly narrower CI. With a much larger data set, we conducted more subgroup analyses, which resulted in more implications.

In the subgroup analysis, we noted a large variety of asymptomatic rates among different populations. For instance, children and adults aged $\leq 45$ years old tended to have a significantly higher rate. The inverse correlation between age and symptom severity has also been reported and discussed by a few studies, and the higher prevalence of chronic diseases among elderies was considered as risk factors for symptomatic infection. ${ }^{232627}$ Infection control measures may be targeted on early detection and isolation of asymptomatic youth, as young asymptomatic carriers are more likely to bring in community transmission due to their more socially active lifestyle habits, with more frequent travelling, compared with people in other age groups. ${ }^{26}$

We also noted that the rate of asymptomatic infections in pregnant women $(48.8 \%)$ was more than two times than in general adults $(20.4 \%)$. A previous study suggested cytotoxic cells may be poised to control virus load and hence affect disease severity during pregnancy. ${ }^{28}$ Case reports from a New York hospital reported a similarly high rate (14 women, $32.6 \%$ ) at presentation, yet $71.4 \%$ (10 women) of the asymptomatic mothers developed symptoms during their hospitalisation and postpartum course. ${ }^{29}$ Nevertheless, we noted that 15 out of the 17 included studies on pregnant women were cross-sectional studies, where the high proportion of asymptomatic infections may include both presymptomatic and asymptomatic cases. More follow-up studies among pregnant women are needed before drawing further conclusions. Undetected asymptomatic pregnant women may lead to more severe consequences. Without early detection and proper preventive measures, the delivery of asymptomatic patients brings extra risks to nosocomial infection and 
may also result in droplet transmission among women, kids and other family members. ${ }^{29}$

To our knowledge, this is the first meta-analysis to compare the rate of asymptomatic infections in different continents. A previous meta-analysis only concluded that populations in Asia may have a lower asymptomatic ratio, while not exploring differences among other continents. ${ }^{30}$ Our results also showed that the estimated ratio in Asia was the lowest among all continents. Possible explanations were summarised in a published meta-analysis, from the perspectives of infection control policies and host characteristics. ${ }^{30}$ Meanwhile, the rate in Africa (64.3\%) was the highest among all continents, although the limited number of subjects (20 271 COVID-19 infections) in African studies reminded us to think twice before generalising the results. The phenomenon may be partly explained by a few factors in African patients, including the generally younger age, the lower proportion of patients and elderlies with chronic diseases living in nursing homes, and the higher serum vitamin D levels due to rich sun exposure. ${ }^{31}$ Despite the higher asymptomatic ratio, it is noted that Africa was the continent with the lowest reverse transcription polymerase chain reaction (RT-PCR) testing rate as well as the lowest vaccination rate. The high rate of asymptomatic infections may further hinder the timely detection and control of COVID-19 outbreaks.

We noted that the rate of asymptomatic infections was nearly doubled in screening studies $(36.0 \%)$ than in nonscreening studies $(19.4 \%)$. One possible explanation is that people may get tested for varied reasons associated with social, cultural and political factors in each region. For instance, front-line health workers and people with a history of exposure were in general more likely to be reported in screening programmes, hence more likely to be detected during the early stage of their infection. Comparatively, the samples in non-screening studies were likely to be dominated by subjects having severe symptoms requiring hospitalisation. ${ }^{32}$ If we consider the rate as a constant, the higher asymptomatic infection rate estimates were likely due to higher probabilities of ascertaining asymptomatic COVID-19 infections in the community with screening implemented. This implies the importance of mass screening in detecting infections, which is important in community infection control. Increasing the accessibility and affordability of community testing could be an important surveillance strategy for early containment of diagnosed cases. ${ }^{33}$

We also found that the pooled asymptomatic infection rate increased from $13.3 \%$ (95\% CI 9.8\% to $17.1 \%$ ) in studies conducted before 1 March 2020 to $42.5 \%$ (33.4\% to $51.9 \%)$ in studies conducted on 1 March 2020 or afterwards, although the $95 \%$ CI is larger in the latter time period. This timeline was highly consistent with a previous study using publicly released data from the Centre for Health Protection in Hong Kong. ${ }^{34}$ The increased rate may be due to overlooking the asymptomatic cases, especially presymptomatic cases, during the early stage of the pandemic, when medical resources were targeted to patients with severe symptoms. Later, with increased public awareness and test accessibility, more COVID-19-infected individuals without symptoms were detected, while more and more studies reported the proportion of asymptomatic patients. Importantly, the SARS-CoV-2 variant with $D$ was replaced by $G$ at the 614 th codon in the spike protein, which has dominated the pandemic since late February 2020, and were suggested to raise the asymptomatic cases. ${ }^{653}{ }^{36}$ Future studies may explore whether the proportion of COVID-19 infections with mild or no symptoms is increasing, especially when considering different SARS-CoV-2 variants.

This study has limitations. First, our pooled asymptomatic infection rates were found to have a high level of heterogeneity $\left(I^{2}=99.7 \%\right)$. This could be attributed to the difficulties in generating the exact number of infections and asymptomatic cases during an outbreak. Although we conducted subgroup analysis and meta-regression to figure out the source of heterogeneity, some unobserved factors which have not been included in the original studies may lead to the high heterogeneity, such as changing pandemic control measures in some countries, the diverse definition of asymptomatic infection, varying practices in surveillance and ascertainment of asymptomatic infection, as well as meteorological disparities across time and regions. Nevertheless, previous studies indicated that any amount of heterogeneity is acceptable if both accurate data and predefined eligibility criteria were provided. ${ }^{37}{ }^{38}$ Second, we followed the WHO definition of COVID-19 infection and only included infections detected by RT-PCR, while the availability of RT-PCR is restricted in many countries. ${ }^{39}$ An increasing number of studies have reported the results of antibody-based rapid diagnostic tests, yet they were not included in our meta-analysis. Third, our meta-analysis focused on real-world evidence and observational studies, where subjects with more severe and easily recognised symptoms were more likely to be selected. The collider bias caused by non-representative sampling strategies (such as sampling conditional on testing and prognosis conditional on hospitalisation) in existing observational studies on COVID-19 has been deeply discussed in Griffith $e t$ $a l^{32}$ Fourth, the cross-sectional studies included in this review might have misclassified presymptomatic cases as 'asymptomatic' and hence resulted in a certain degree of overestimation of the rate of asymptomatic infections. With additional information on the exposure and reporting dates of each case, we remark our estimation can be extended to a right censoring version to further address some potential bias in existing frameworks. ${ }^{12}$

\section{CONCLUSIONS}

We estimated the rate of asymptomatic infections at $23.6 \%$, which decreased to $21.7 \%$ after excluding presymptomatic cases. Subgroup analysis indicated that pregnant women, children, African residents, screening programmes and studies conducted after 1 March 2020 had higher rates of asymptomatic infections. Our findings 
provide further insights on the distribution of asymptomatic COVID-19 infections in different groups of individuals, bearing significance on public health policies that aim to achieve early identification and more stringent containment of the pandemic.

\section{Author affiliations}

${ }^{1}$ School of Public Health, Zhejiang University, Hangzhou, China

${ }^{2}$ Mianyang Maternal and Child Health Care Hospital, Mianyang, China

${ }^{3} \mathrm{JC}$ School of Public Health and Primary Care, The Chinese University of Hong Kong, Hong Kong, China

${ }^{4}$ Department of Applied Mathematics, Hong Kong Polytechnic University, Hong Kong, China

Acknowledgements We thank Ms Maggie Choi, librarian from Li Ping Library, The Chinese University of Hong Kong, for her valuable help in developing our search strategy.

Contributors $\mathrm{XC}, \mathrm{ZH}$, and JW were responsible for the overall study coordination. $\mathrm{XC}$ and $\mathrm{ZH}$ designed the study and searched the literature. $\mathrm{XC}, \mathrm{ZH}$ and JW extracted the data and evaluated the quality. $\mathrm{XC}$ conducted the statistical analysis. $\mathrm{XC}, \mathrm{ZH}$ and JW wrote the full manuscript. SZ, MC-SW, KCC, DH and JL provided critical revision to the manuscript for important intellectual content. All authors discussed the results and approved the final version of the manuscript for publication.

Funding This study was funded by The Key Project of Medicine Discipline of Guangzhou (2017-2019-07). DH was supported by General Research Fund (15205119) of Research Grants Council of Hong Kong.

Disclaimer The funding agencies had no role in the design and conduct of the study; collection, management, analysis, and interpretation of the data; preparation, review, or approval of the manuscript; or decision to submit the manuscript for publication.

Competing interests DH received a grant from Alibaba (China) Collaborative Research Grant. Other authors declared no conflict of interest.

Patient consent for publication Not required.

Provenance and peer review Not commissioned; externally peer reviewed.

Data availability statement All data relevant to the study are included in the article or uploaded as supplementary information. Not applicable.

Supplemental material This content has been supplied by the author(s). It has not been vetted by BMJ Publishing Group Limited (BMJ) and may not have been peer-reviewed. Any opinions or recommendations discussed are solely those of the author(s) and are not endorsed by BMJ. BMJ disclaims all liability and responsibility arising from any reliance placed on the content. Where the content includes any translated material, BMJ does not warrant the accuracy and reliability of the translations (including but not limited to local regulations, clinical guidelines, terminology, drug names and drug dosages), and is not responsible for any error and/or omissions arising from translation and adaptation or otherwise.

Open access This is an open access article distributed in accordance with the Creative Commons Attribution Non Commercial (CC BY-NC 4.0) license, which permits others to distribute, remix, adapt, build upon this work non-commercially, and license their derivative works on different terms, provided the original work is properly cited, appropriate credit is given, any changes made indicated, and the use is non-commercial. See: http://creativecommons.org/licenses/by-nc/4.0/.

Author note $\mathrm{XC}, \mathrm{ZH}$, and JW contributed equally, and are joint first authors.

ORCID iDs

Jingxuan Wang http://orcid.org/0000-0002-1570-7266

Ka Chun Chong http://orcid.org/0000-0001-5610-1298

Daihai He http://orcid.org/0000-0003-3253-654X

Jinhui Li http://orcid.org/0000-0002-2572-3454

\section{REFERENCES}

1 Chan JF-W, Yuan S, Kok K-H, et al. A familial cluster of pneumonia associated with the 2019 novel coronavirus indicating personto-person transmission: a study of a family cluster. Lancet 2020;395:514-23.
2 Team EE. Note from the editors: World Health organization declares novel coronavirus (2019-nCoV) sixth public health emergency of international concern. Eurosurveillance 2020;25:200131e.

3 Zhao S, Lin Q, Ran J, et al. Preliminary estimation of the basic reproduction number of novel coronavirus (2019-nCoV) in China, from 2019 to 2020: a data-driven analysis in the early phase of the outbreak. Int J Infect Dis 2020;92:214-7.

4 Zou L, Ruan F, Huang M, et al. SARS-CoV-2 viral load in upper respiratory specimens of infected patients. $N$ Engl J Med 2020;382:1177-9.

5 Lee S, Kim T, Lee E, et al. Clinical course and molecular viral shedding among asymptomatic and symptomatic patients with SARS-CoV-2 infection in a community treatment center in the Republic of Korea. JAMA Intern Med 2020;180:1447-52.

6 Chen X, Pan Z, Yue S, et al. Disease severity dictates SARS-CoV2 -specific neutralizing antibody responses in COVID-19. Signal Transduct Target Ther 2020;5:180.

7 Bai Y, Yao L, Wei T, et al. Presumed asymptomatic carrier transmission of COVID-19. JAMA 2020;323:1406-7.

8 Hoehl S, Rabenau H, Berger A, et al. Evidence of SARS-CoV-2 infection in returning travelers from Wuhan, China. $N$ Engl $J$ Med 2020;382:1278-80.

9 Pan Y, Yu X, Du X, et al. Epidemiological and clinical characteristics of 26 asymptomatic severe acute respiratory syndrome coronavirus 2 carriers. J Infect Dis 2020;221:1940-7.

10 Lauer SA, Grantz KH, Bi Q, et al. The incubation period of coronavirus disease 2019 (COVID-19) from publicly reported confirmed cases: estimation and application. Ann Intern Med 2020;172:577-82.

11 Chen Y, Wang AH, Yi B, et al. Epidemiological characteristics of infection in COVID-19 close contacts in Ningbo city. Zhonghua Liu Xing Bing Xue Za Zhi 2020;41:667-71.

12 Mizumoto K, Kagaya K, Zarebski A, et al. Estimating the asymptomatic proportion of coronavirus disease 2019 (COVID-19) cases on board the diamond Princess cruise SHIP, Yokohama, Japan, 2020. Eurosurveillance 2020;25:2000180.

13 Gao W, LM L. Research progress on the incubation period of new coronavirus pneumonia or the transmission of recessive infections. Chinese Journal of Epidemiology 2020;41:485-8.

14 Moher D, Liberati A, Tetzlaff J, et al. Preferred reporting items for systematic reviews and meta-analyses: the PRISMA statement. PLoS Med 2009;6:e1000097.

15 Kronbichler A, Kresse D, Yoon S, et al. Asymptomatic patients as a source of COVID-19 infections: a systematic review and metaanalysis. Int $J$ Infect Dis 2020;98:180-6.

16 The People's Government of Beijing Municipality. Notice of the state Council on the joint prevention and control mechanism for the prevention and control of the novel coronavirus infection and pneumonia epidemic on Issuing the regulations for the management of asymptomatic patients with the new coronavirus, 2020. Available: http://www.beijing.gov.cn/zhengce/zhengcefagui/202004/ t20200409 1798158.html

17 World Health Organization (WHO). COVID-19 clinical management: living guidance, 2020. Available: https://www.who.int/publications/i/ item/WHO-2019-nCoV-clinical-2021-1

18 Downes MJ, Brennan ML, Williams HC, et al. Development of a critical appraisal tool to assess the quality of cross-sectional studies (axis). BMJ Open 2016;6:e011458.

19 Marsh A, Eslick EM, Eslick GD. Does a diet low in FODMAPs reduce symptoms associated with functional gastrointestinal disorders? A comprehensive systematic review and meta-analysis. Eur J Nutr 2016;55:897-906.

20 Duval S, Tweedie R. Trim and fill: a simple funnel-plot-based method of testing and adjusting for publication bias in meta-analysis. Biometrics 2000;56:455-63.

$21 \mathrm{Ji} \mathrm{T}$, Chen H-L, Xu J, et al. Lockdown contained the spread of 2019 novel coronavirus disease in Huangshi City, China: early epidemiological findings. Clin Infect Dis 2020;71:1454-60.

22 Grados IZ, Paredes RL, Benavides G. Characteristics of SARSCoV-2 infection in pregnant and puerperal women at Callao national Hospital. Rev Peru Ginecol Obstet 2020;66.

23 Centers for Desease Control and Prevention. Interim clinical guidance for management of patients with confirmed coronavirus disease (COVID-19), 2020. Available: https://www.cdc.gov/ coronavirus/2019-ncov/hcp/clinical-guidance-management-patients. html

24 Byambasuren O, Cardona M, Bell K, et al. Estimating the extent of asymptomatic COVID-19 and its potential for community transmission: systematic review and meta-analysis. JAAMI 2020;5:223-34. 
25 Cai J, Sun W, Huang J, et al. Clinical features and the treatment of children with COVID-19: a case series from Wenzhou, China. J Med Virol 2020;92:2403-5.

26 Zhang S, Xu Y, Li J, et al. Symptomless multi-variable apnea prediction index assesses adverse outcomes in patients with corona virus disease 2019. Sleep Med 2020;75:294-300.

27 Du W, Yu J, Wang H, et al. Clinical characteristics of COVID-19 in children compared with adults in Shandong Province, China. Infection 2020;48:445-52.

28 Edlow AG, Li JZ, Collier A-RY, et al. Assessment of maternal and neonatal SARS-CoV-2 viral load, transplacental antibody transfer, and placental pathology in pregnancies during the COVID-19 pandemic. JAMA Netw Open 2020;3:e2030455-e.

29 Breslin N, Baptiste C, Gyamfi-Bannerman C, et al. Coronavirus disease 2019 infection among asymptomatic and symptomatic pregnant women: two weeks of confirmed presentations to an affiliated pair of New York City hospitals. Am J Obstet Gynecol MFM 2020;2:100118.

30 Chen C, Zhu C, Yan D, et al. The epidemiological and radiographical characteristics of asymptomatic infections with the novel coronavirus (COVID-19): a systematic review and meta-analysis. Int J Infect Dis 2021;104:458-64.

31 Bamgboye EL, Omiye JA, Afolaranmi OJ. COVID-19 pandemic: is Africa different? J Natl Med Assoc 2021;113:324-35.
32 Griffith GJ, Morris TT, Tudball MJ, et al. Collider bias undermines our understanding of COVID-19 disease risk and severity. Nat Commun 2020;11:5749.

33 Padula WV. Why only test symptomatic patients? consider random screening for COVID-19. Appl Health Econ Health Policy 2020;18:333-4.

34 Tao J, Zhang X, Zhang X, et al. The time serial distribution and influencing factors of asymptomatic COVID-19 cases in Hong Kong. One Health 2020;10:100166.

35 Korber B, Fischer WM, Gnanakaran S, et al. Tracking changes in SARS-CoV-2 spike: evidence that D614G increases infectivity of the COVID-19 virus. Cell 2020;182:e19:812-27.

36 Meng F, Xu R, Wang S, et al. Human umbilical cord-derived mesenchymal stem cell therapy in patients with COVID-19: a phase clinical trial. Signal Transduct Target Ther 2020;5:172.

37 Higgins JPT. Commentary: heterogeneity in meta-analysis should be expected and appropriately quantified. Int $J$ Epidemiol 2008;37:1158-60.

38 Zhu J, Ji P, Pang J, et al. Clinical characteristics of 3062 COVID-19 patients: a meta-analysis. J Med Virol 2020;92:1902-14.

39 Boum Y, Fai KN, Nikolay B, et al. Performance and operational feasibility of antigen and antibody rapid diagnostic tests for COVID-19 in symptomatic and asymptomatic patients in Cameroon: a clinical, prospective, diagnostic accuracy study. Lancet Infect Dis 2021;21:1089-96. 